\title{
Reglas de despacho en la programación de procedimientos quirúrgicos electivos: impacto en los indicadores de ocupación y oportunidad
}

\author{
Dispatching Rules in Elective Surgery Scheduling: Impact on Service Occupation and Opportunity \\ Regras de despacho na programação de procedimentos cirúrgicos eletivos: impacto nos indicadores de \\ ocupação e oportunidade \\ Ana M. Estupiñán BSc. ', M. Juliana Torres BSc. ', Martha P. Caro MSc. ', Eliana María González-Neira MSc. ', \\ David Barrera MSc. ', Nicolás Pérez MD², Jorge Barbosa MD², Carlos Sefair MD², Daniel R. Suárez PhD' \\ Recibido: 29 de mayo de $2015 \bullet$ Aceptado: 25 de enero de 2016 \\ Doi: dx.doi.org/10.12804/revsalud14.02.2016.06 \\ Para citar este artículo: Estupiñán AM, Torres MJ, Caro MP, González-Neira EM, Barrera D, Pérez N, et al. Reglas de despacho en la programación de procedimientos quirúr- \\ gicos electivos: impacto en los indicadores de ocupación y oportunidad. Rev Cienc Salud. 2016;14(2):21 1-21. doi: dx.doi.org/10.12804/revsalud14.02.2016.06
}

\section{Resumen}

Introducción: la programación de los quirófanos es el factor de mayor incidencia en el desempeño de los servicios de cirugías. Este estudio cuantificó el impacto de la variabilidad artificial creada por una programación manual de las cirugías en el Hospital Universitario Mayor - Méderi (HUM), Colombia. La hipótesis planteada es que una programación semiautomática podría: (i) reducir la variabilidad diaria del servicio de cirugías, (ii) aumentar la disponibilidad de los quirófanos y (iii) mejorar el tiempo de oportunidad por cirugía. Materiales y métodos: este estudio empleó los registros del servicio de cirugía de un mes regular. El servicio estudiado ejecuta alrededor de 35000 cirugías al año y el proceso de programación es manual. La programación real fue comparada con las generadas a partir del empleo del algoritmo Bin Packing y las reglas de despacho Longest Processing Time (LPT) y Shortest Processing Time (SPT). Resultados: la aplicación del algoritmo con la regla LPT logró una mejora en la programación del mes estudiado: los coeficientes de variación del flujo de pacientes y ocupación diaria se redujeron (25,09 \% y 36,71 \%, respectivamente). Adicionalmente, el tiempo de oportunidad se redujo en 6,2 días y la ocupación del servicio subió un 26,22 \%. La programación con la regla SPT aumentó la variabilidad en el flujo de paciente en 22,7 \% y disminuyó la ocupación en 2,28 \%. Conclusiones: una programación semiautomática de las salas de cirugía en el HUM

\footnotetext{
1 Departamento de Ingeniería Industrial, Facultad de Ingeniería, Pontificia Universidad Javeriana, Bogotá, Colombia.

2 Hospital Universitario Mayor - Méderi, Colombia.
} 
empleando la regla LPT lograría mejorar sustancialmente indicadores de variabilidad del servicio, tiempo de oportunidad y ocupación.

Palabras clave: salas de cirugía, algoritmos, programación.

\section{Abstract}

Introduction: The performance of a surgery service is highly impacted by its schedule. This study measured the impact of the artificial variability caused by a manual scheduling of surgeries at "Hospital Universitario Mayor - Méderi (HUM)" (Colombia). The hypotheses were that the proposed algorithm is able to (i) reduce daily service variation, (ii) increase the availability of service resources, and (iii) improve the opportunity time for each surgery. Materials and methods: The studied surgical service performs around 35000 annual surgeries and its scheduling process is presently manually made. Actual scheduling records of a regular month were compared to schedules generated by a Bin Packing (BP) algorithm hybridized with the Longest Processing Time (LPT) and Shortest Processing Time (SPT) dispatching rules. Results: It was found that the BP algorithm with LPT rule could improve service performance, reducing the variation coefficients of patients' flow and daily service occupation by $25.09 \%$ and $36.71 \%$, respectively. The programmed surgeries were also moved ahead 6.2 days, and the overall occupation rate increased by $26.72 \%$. Results were not better when a SPT rule was used, boosting the variability on patient flow by $22.7 \%$ and reducing the occupation by $2.28 \%$. Conclusions: Semiautomatic scheduling of the surgical service at the HUM, a BP algorithm with LPT rule, may substantially increase service performance in terms of service occupation and opportunity.

Keywords: Operating rooms, Algorithms, Programming.

\section{Resumo}

Introdução: A programação das salas de cirurgia é o fator de maior incidência no desempenho dos serviços de cirurgias. Este estudo quantificou o impacto da variabilidade artificial criada por uma programação manual das cirurgias no Hospital Universitário Mayor - Méderi (HUM), Colômbia. A hipótese apresentada é que uma programação semiautomática poderia: (i) reduzir a variabilidade diária do serviço de cirurgias, (ii) aumentar a disponibilidade das salas de cirurgia, e (iii) melhorar o tempo de oportunidade por cirurgia. Materiais e métodos: Este estudo empregou os registros do serviço de cirurgia de um mês regular. O serviço estudado executa cerca de 35000 cirurgias por ano e o processo de programação é manual. A programação real foi comparada com as geradas a partir do emprego do algoritmo Bin Packing e as regras de despacho Longest Processing Time (LPT) e Shortest Processing Time (SPT). Resultados: A aplicação do algoritmo com a regra LPT conseguiu uma melhora na programação do mês estudado: os coeficientes de variação do fluxo de pacientes e ocupação diária reduziram-se (25,09 \% y 36,71 \%, respectivamente). Adicionalmente, o tempo de oportunidade reduziu-se em 6,2 dias e a ocupação do serviço subiu um $26,22 \%$. A programação com a regra SPT aumentou a variabilidade no fluxo de paciente em 22,7 \% e diminuiu a ocupação em 2,28 \%. Conclusões: Uma programação semiautomática das salas de cirurgia no HUM empregando a regra LPT conseguiria melhorar substancialmente indicadores de variabilidade do serviço, tempo de oportunidade e ocupação.

Palavras-chave: Salas de Cirurgia, Algoritmos, Programação. 


\section{Introducción}

El sector salud enfrenta nuevos retos debido a la demanda de una mayor cobertura y una mejor calidad del servicio cuando, a la vez, está expuesto a continuas reducciones en su presupuesto. Los hospitales no son ajenos a esta problemática. En Europa, por ejemplo, la demanda hospitalaria creció $6,5 \%$ en 2010 y el presupuesto no creció más del $2 \%$ en los años siguientes, creando un estrés económico en los hospitales públicos (1). En consecuencia, la investigación de metodologías para asignación de recursos ha crecido de manera sostenida (2).

Particularmente, el departamento de cirugía de un hospital es importante tanto para la misión de este como para su viabilidad económica. El servicio de cirugías genera alrededor del $40 \%$ de las ganancias totales en un hospital promedio y, a la vez, es el departamento más costoso, debido a que representa, aproximadamente, el $9 \%$ del presupuesto anual del hospital $(3,4)$. Esto hace que el optimizar los recursos pertenecientes al servicio de cirugías sea esencial para la viabilidad económica del hospital. Más aún, revisiones recientes han encontrado que los servicios quirúrgicos concentran parte importante de la investigación orientada a optimizar recursos en los hospitales $(5,6)$.

Ahora bien, existen diversidad de elementos que influyen en el desempeño y eficiencia de las salas de cirugía: cancelaciones de último minuto, complicaciones en el quirófano, horas extra innecesarias de personal médico, entre otros. Todo lo cual puede llegar a traducirse en mayores costos, retrasos y operaciones que presentan una duración mayor a la estimada (7). Se sabe que la variabilidad de los procesos va en contra de su eficiencia, por lo que es fundamental que los hospitales se empeñen en disminuir la variabilidad del servicio de cirugías para así aumentar la rentabilidad total que aporta al hospital (7).
En particular, se ha identificado que una programación deficiente de las cirugías es el factor de mayor incidencia en el desempeño y rendimiento del servicio de cirugías de un hospital (8). Una mala programación del servicio de cirugías, debido al error humano o a políticas poco claras, puede introducir variabilidad en el sistema, lo que atenta contra la calidad del servicio e incrementa los costos. Este tipo de variabilidad ha sido clasificado como artificial, ya que no es inherente al proceso (i.e. la cirugía) y puede ser eliminada completamente por medio de una gestión y planeación adecuada (9). Sin embargo, a pesar de la relevancia y el aumento de las investigaciones en programación de servicios quirúrgicos, existe todavía una brecha con las prácticas de los hospitales (10). A nivel internacional, uno de los principales retos de la comunidad investigativa es lograr espacios de colaboración que permitan mayor impacto de los desarrollos en sistemas reales $(11,12)$.

En Colombia, el escenario es similar. Si bien, existen estudios aislados de programación o planeación de los servicios quirúrgicos, estos se encuentran en una etapa temprana de desarrollo, comparados con la investigación internacional (13-16). Más aún, de acuerdo con Velasco et al., se debe profundizar en el trabajo colaborativo entre académicos y hospitales (17). Para que el proceso sea exitoso, es necesario que las investigaciones sean producto de la relación entre administradores del sistema e investigadores (18).

El Hospital Universitario Mayor - Méderi (HUM) es uno de los centros médicos de mayor tamaño y complejidad de la cuidad de Bogotá, y no está exento del problema anteriormente descrito. En la actualidad, el Hum cuenta con 19 quirófanos y ejecuta, en promedio, 36000 cirugías al año. Al momento de realizar este estudio, se encontró que el proceso de programación de cirugías es manual y con el paso 
del tiempo se vuelve cada vez más complejo y propenso al error. La tasa de ocupación promedio del servicio es actualmente del 51,06 \% . La meta establecida por los directivos es del $86 \%$, ya que una tasa de ocupación mayor podría afectar la atención de pacientes que ingresan por el servicio de urgencias. Por otra parte, el tiempo que transcurre desde la solicitud de una sala de cirugía hasta el momento que efectúa el procedimiento — tiempo de oportunidadpuede ser superior a un mes e impacta directamente en la satisfacción y calidad del servicio.

Este trabajo se enfoca en cuantificar el impacto de la variabilidad artificial creada por una programación manual de las cirugías y en evaluar diferentes estrategias para eliminarla y/o disminuirla. Para ello, se comparó el método actual de programación contra la programación lograda a partir de la aplicación de un algoritmo que usa diferentes reglas de despacho. Se presentan las hipótesis de que una programación semiautomática puede (i) reducir la variabilidad del servicio de cirugías, (ii) aumentar la disponibilidad de los recursos del servicio y (iii) mejorar el tiempo de oportunidad para una cirugía.

\section{Materiales y métodos}

Este estudio es descriptivo y cuantitativo. La metodología abarcó tres fases principales: el diagnóstico del servicio, el desarrollo de alternativas para efectuar la programación y la validación de los resultados encontrados mediante los indicadores propuestos. Para la ejecución, se recolectó la información de todas las cirugías efectuadas durante mayo de 2014. Dado que el mes fue considerado como normal por las directivas del servicio, el tamaño de muestra seleccionado permite concluir sobre el funcionamiento de este $(19,20)$. De los registros históricos se obtuvieron duraciones estimadas para cada procedimiento, hora de inicio y hora finalización. Las cirugías con errores en el registro del servicio, por ejemplo, información incompleta o errónea, fueron excluidas. Debido a la naturaleza del estudio, no fue necesario acceder a información confidencial de los pacientes o historias clínicas.

Diagnóstico del servicio de cirugías: el servicio de cirugías del hum cuenta con 19 quirófanos heterogéneos, esto es, no todas las operaciones pueden efectuarse en cualquier quirófano. En la actualidad, no existen franjas horarias destinadas a especialidades particulares. Esto implica que, si un quirófano cuenta con los recursos necesarios para una operación, esta última puede ser programada sin restricciones de fecha u horario. Finalmente, el horizonte de planeación es de una semana y el tiempo de oportunidad es cercano a un mes. En consecuencia, el sistema puede ser modelado usando programación offline, sin que esto implique sacrificios en el nivel de servicio.

Para diagnosticar el funcionamiento del sistema, con los registros históricos del hospital, se evaluó (i) la tasa de ocupación promedio $(\overline{T Q})$, medida como la relación entre el tiempo ocupado y el disponible; y (ii) la variabilidad del sistema, medida por medio de los coeficientes de variación diarios para el flujo de pacientes y la ocupación.

Alternativas para efectuar la programación de cirugías: dado que el objeto principal de la investigación es determinar el impacto de la variabilidad artificial causada por la programación manual, se seleccionó un algoritmo de programación semiautomática heurístico. El objetivo es que la mejora esté explicada por la eliminación de la variabilidad artificial y no por la sofisticación del método. En particular, se probó el algoritmo Bin Packing propuesto por Dexter et al. en combinación con una de dos reglas de despacho: Longest Processing Time (LPT) o Shortest Processing Time (SPT) (21). El Bin-Packing es útil cuando las salas de cirugía tienen características específicas que aplican solo a ciertos 
tipos o especialidades quirúrgicas. Por su parte, las reglas de despacho han sido seleccionadas por el impacto logrado en estudios anteriores. Por ejemplo, LPT tiene el objetivo de impactar la tasa de ocupación y disminuir el tiempo total del proceso (22); y SPT tiene el objeto de disminuir los tiempos de flujo del proceso (23). La combinación de Bin-Paking y heurísticas básicas ha sido probada exitosamente en trabajos recientes $(24,25)$.

En ese orden de ideas, para las programaciones semiautomáticas las cirugías se organizan en función de su duración estimada de acuerdo con la regla de despacho seleccionada. Posteriormente, se asigna a la primera cirugía en la lista a la fecha y horario más temprano posible en el que se encuentre un quirófano que sea apto para el procedimiento y que posea la disponibilidad de tiempo suficiente (figura 1). En caso de que el tiempo del procedimiento exceda el tiempo libre de las salas disponibles, la cirugía pasa a una cola de espera. Al cambiar de día, el algoritmo procede a secuenciar inicialmente los casos de la cola de espera y luego los casos nuevos. Este funcionamiento se ilustra con un ejemplo de 8 cirugías ordenadas con la regla SPT en la figura 2.

La pertinencia del algoritmo propuesto se sustenta en los siguientes cinco supuestos: (i) la programación es offline, esto es, se conoce el listado de procedimientos a ser programados desde el inicio del horizonte de planeación; (ii) la duración de la cirugía incluye los tiempos de alistamiento y recambio entre salas; (iii) la programación se realiza para una semana de siete días; (iv) se asume que siempre hay recursos disponibles por quirófano para todas las cirugías y (v) la ocupación máxima del servicio es $86 \%$ de acuerdo con la estrategia empleada por el servicio de cirugías estudiado.

Validación del algoritmo y sus beneficios: para la evaluación del algoritmo propuesto se establecieron los siguientes tres diferentes escenarios: (i) escenario actual: programación real elaborada por el servicio de cirugías del Hospital, (ii) escenario SPT: programación generada por el algoritmo empleando la regla SPT; y (iii) escenario LPT: programación generada por el algoritmo empleando la regla $L P T$.

Las programaciones de los tres escenarios fueron comparadas en términos de la tasa de ocupación promedio y los coeficientes de variación diaria para el número de pacientes y la ocupación. Adicionalmente, para los escenarios SPT y LPT se evaluó el cambio en el tiempo de oportunidad por cirugía $(\Delta \overline{T Q})$, en número de días, con respecto a la programación manual.

\section{Resultados}

Porcentaje de ocupación diaria promedio: el algoritmo propuesto con las dos reglas de despacho logró una mejoría en cuanto a la distribución de las cirugías, inclusive con la restricción de solamente alcanzar una tasa de ocupación máxima del $86 \%$. El escenario más atractivo según este indicador es el de $L P T$, pues logra alcanzar una tasa de ocupación del 77,28 \% empleando tan solo 19 días para la programación de las cirugías.

Es necesario aclarar que para el escenario $S P T$, a pesar de programar las cirugías en los mismos 30 días que lo hace el Hospital, y teniendo una tasa de ocupación muy similar, existe una gran diferencia, y es que este escenario está reduciendo la subutilización de los recursos, pues en los primeros 19 días se logra una tasa de ocupación del 72,7 \% . Sin embargo, debido a que los días restantes su tasa de ocupación baja significativamente a 5,8 \%, ya que se dejan a disponibilidad 9 de las 10 salas disponibles, la tasa de ocupación sufre una alteración (se disminuye), lo que da origen al 48,78\% de ocupación promedio presentado anteriormente. 


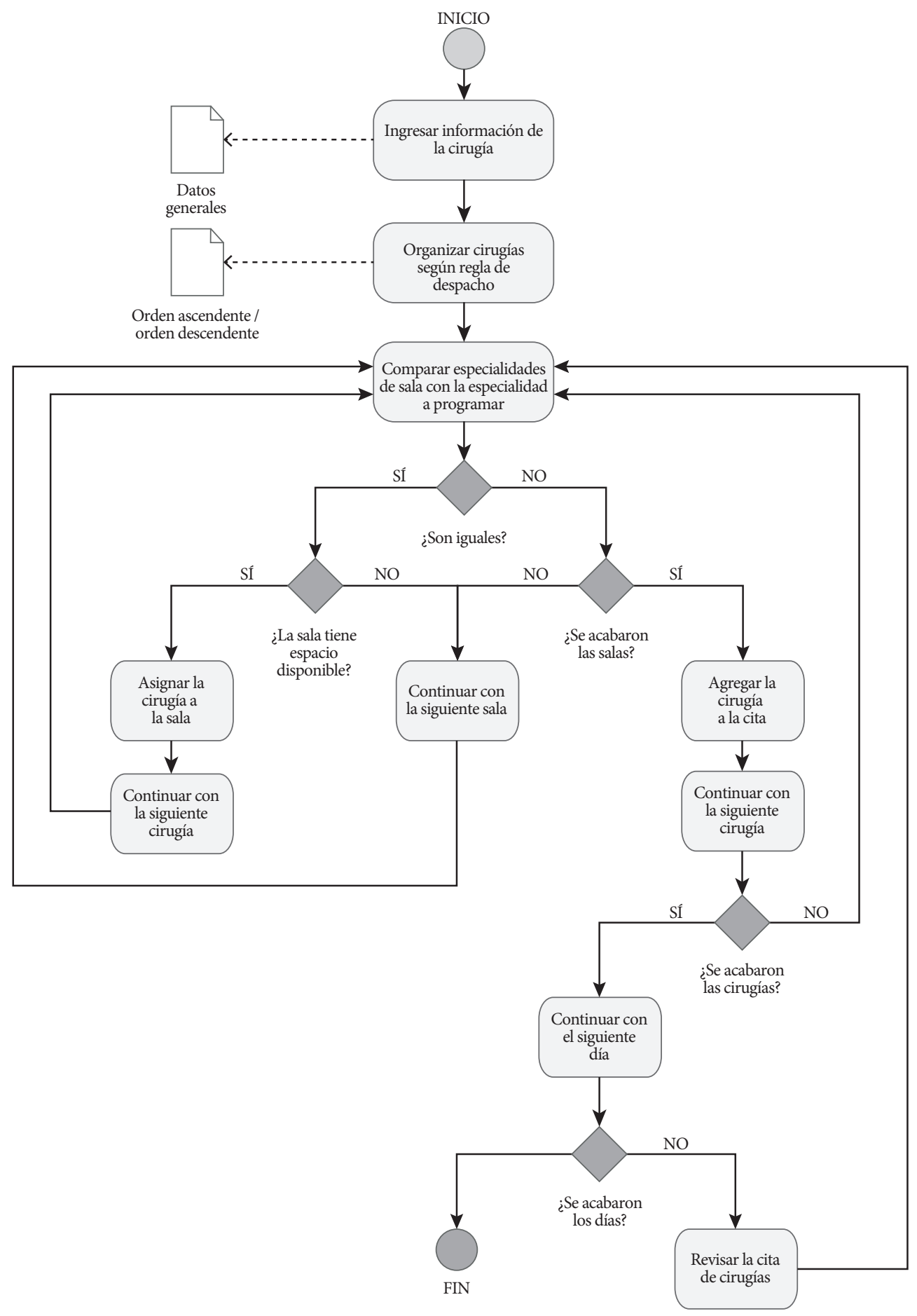

Figura 1. Descripción general del algoritmo de programación semiautomático 


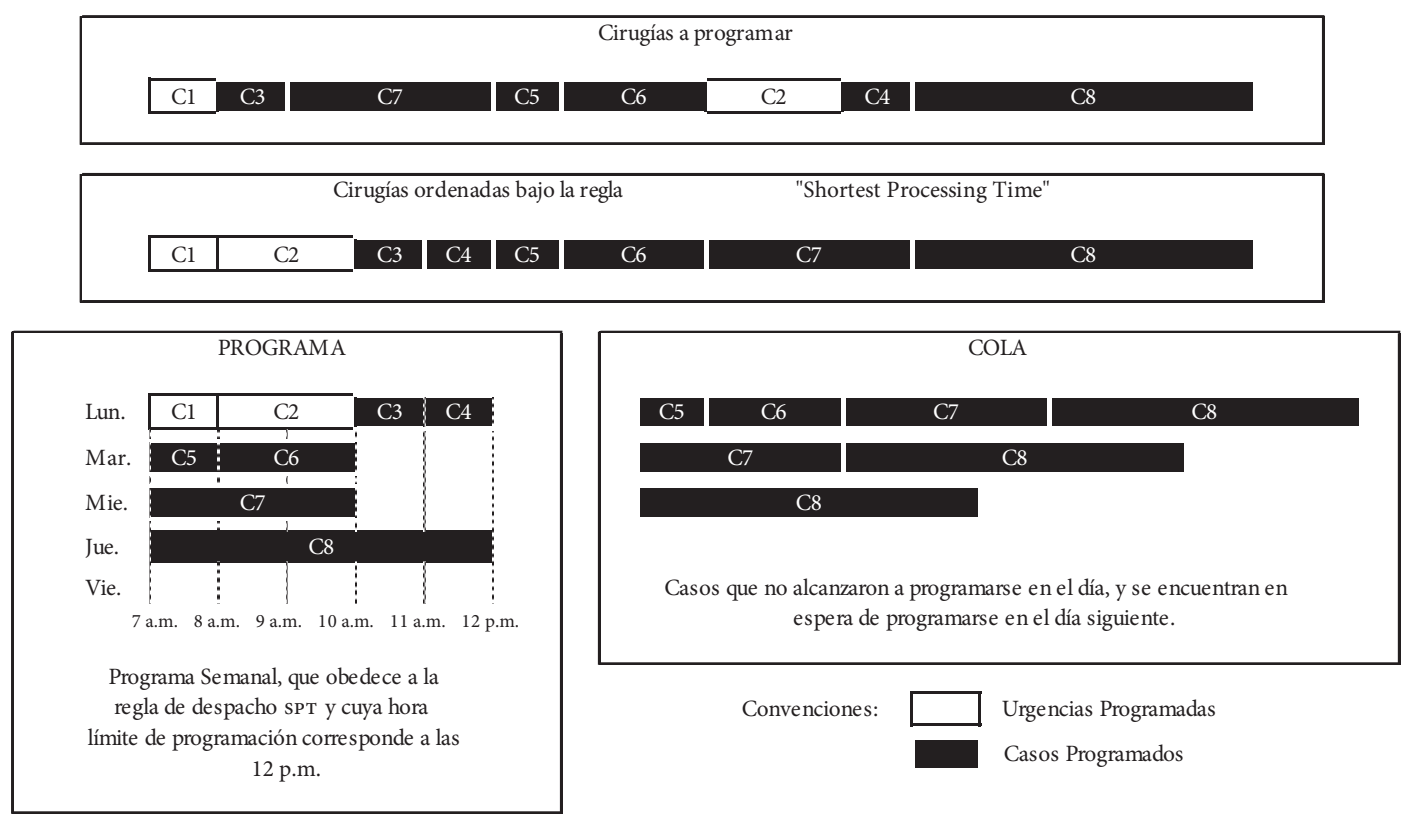

Figura 2. Ejemplo ilustrativo del algoritmo bajo la regla de despacho sPt para una sala

Fuente: elaboración propia con base en Blake y Donald (26)

Coeficiente de variabilidad del flujo de pacientes: en la figura 3 se presenta el comportamiento del coeficiente de variación del flujo de pacientes programados. En el escenario LPT se observa un cambio brusco en el día 20, pues a partir de ese día y hasta el fin del mes no se programó ninguna cirugía. Esto se debe a que el algoritmo logró programar la totalidad de las cirugías en un intervalo de tiempo menor al estimado por el Hospital. Por otro lado, el escenario SPT tiene una tendencia que va disminuyendo desde el día 13, ya que desde ese día solamente se tiene la programación de cirugías de especialidades con duraciones largas, para las cuales solo existe una sala. Esto hace que se extienda el número de días para la programación y que el coeficiente de variación se vea afectado, pues las demás salas no tienen ninguna programación pendiente.

Teniendo en cuenta el comportamiento de los escenarios, con respecto al coeficiente de varia- ción de flujo de pacientes, se logra establecer que el escenario LPT es el que tiene menor variabilidad al disminuir el coeficiente de variación actual en un $25,09 \%$.

Coeficiente de variabilidad de la ocupación del servicio: para el caso del coeficiente de variación de la ocupación del servicio, se acotó el análisis a los primeros 19 días de programación de los tres escenarios, basándose en los resultados obtenidos en el coeficiente de variación en el flujo de pacientes. Esto se realizó con el fin de comparar el periodo en el que se programa aproximadamente el 98,26\% de las cirugías para los escenarios LPT y SPT, lo que evita que la falta de cirugías a programar al final del mes afecte los resultados de los coeficientes de variación (figura 4).

Con base en esto, se logra identificar que la regla de despacho SPT es la que presenta un menor coeficiente de variación con respecto a los demás escenarios. Sin embargo, la regla de 


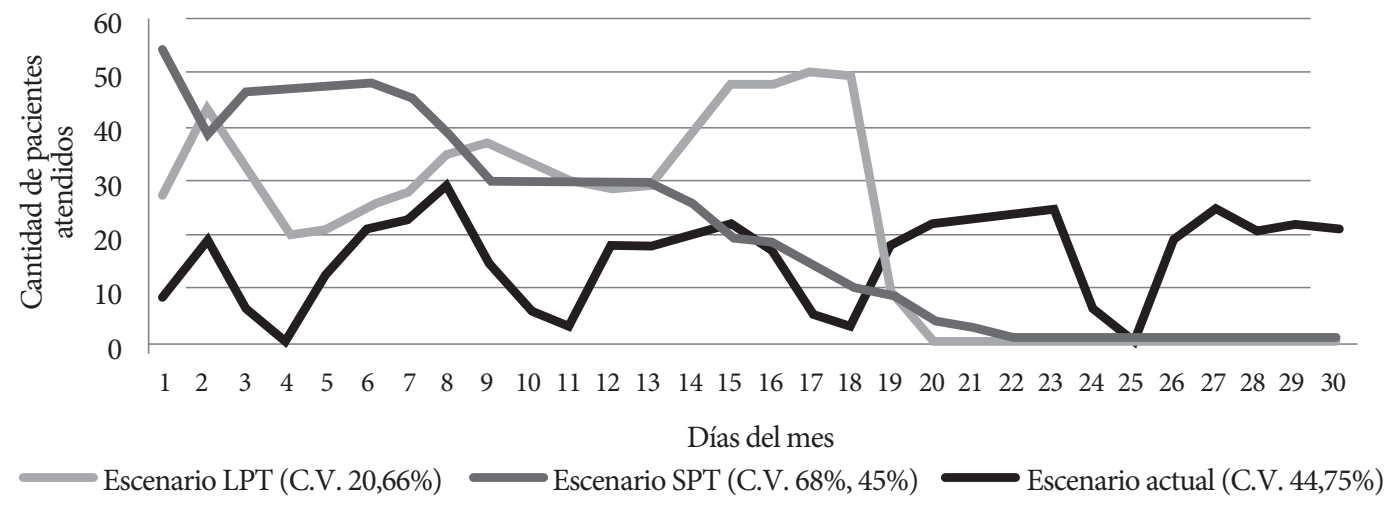

Figura 3. Comportamiento del flujo de pacientes (en términos de frecuencia) para la programación actual y los escenarios simulados SPT y LPT

despacho $L P T$ le sigue con un coeficiente un $5 \%$ mayor, que sigue siendo un 36,71\% menor al coeficiente de la programación actual del Hospital (C.V. del 57,37\%), logrando uno de los objetivos planteados en el proyecto.

Cambio en el tiempo de oportunidad con respecto a la programación (escenario) actual: en la tabla 1 se puede apreciar que ambas reglas de despacho logran adelantar en más de 6 días en promedio la programación de las cirugías, siendo la regla SPT la mejor opción. Esto quiere decir que el tiempo transcurrido entre la solicitud de un paciente para su programación y el momento en donde será efectuada la cirugía se lograría reducir drásticamente, apuntando a una mejora en el tiempo de espera por parte de los pacientes y así, satisfaciendo una de las necesidades principales que posee el Hum.

\section{Discusión}

En este trabajo se encontró que una programación semiautomática, basada en el algoritmo Bin-Packing y reglas de despacho, aumenta la disponibilidad de los recursos y reduce la variabilidad

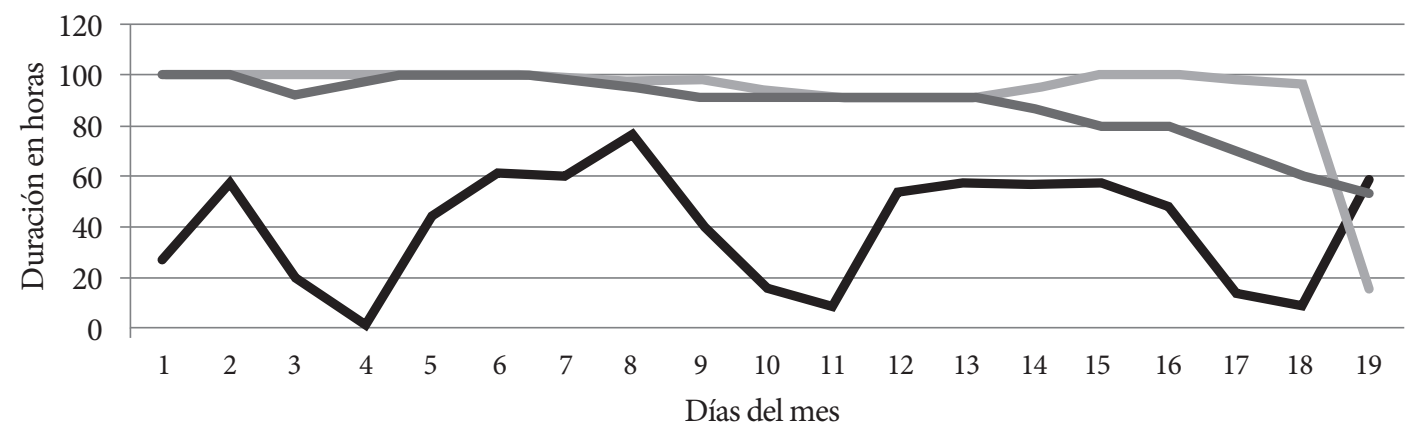

$\longrightarrow$ Escenario LPT (C.V. 20,66\%) $\longrightarrow$ Escenario SPT (C.V. 68\%, 45\%) $\longrightarrow$ Escenario actual (C.V. 44,75\%)

Figura 4. Comportamiento de la ocupación del servicio de cirugías hasta el día 19 del mes, para la programación actual y los escenarios simulados SPT y LPT 
Reglas de despacho en la programación de procedimientos quirúrgicos electivos: impacto en los indicadores de ocupación y oportunidad

Tabla 1. Resultados de los indicadores elegidos para evaluar los diferentes escenarios de programación de cirugías para el del mes de mayo de 2014

\begin{tabular}{|c|c|c|c|c|c|}
\hline \multirow{2}{*}{$\begin{array}{c}\text { Escenario de } \\
\text { análisis }\end{array}$} & \multirow{2}{*}{$\begin{array}{c}\text { Tasa de } \\
\text { ocupación } \\
\text { promedio } \\
(\overline{T Q})\end{array}$} & \multicolumn{2}{|c|}{ Coeficientes de variación } & \multirow{2}{*}{$\begin{array}{c}\text { Cambio en } \\
\text { el tiempo de } \\
\text { oportunidad } \\
\text { promedio } \\
(\Delta \overline{T Q})\end{array}$} & \multirow{2}{*}{ Días programados } \\
\hline & & Pacientes & Ocupación & & \\
\hline S. Actual & $51,06 \%$ & $45,75 \%$ & $57,37 \%$ & $\mathrm{~N} / \mathrm{A}$ & 30 días \\
\hline SPT & $48,78 \%$ & $68,45 \%$ & $15,58 \%$ & 8,5 días antes & 30 días \\
\hline LPT & $77,28 \%$ & $20,66 \%$ & $20,66 \%$ & 6,2 días antes & 19 días \\
\hline
\end{tabular}

artificial del servicio quirúrgico. Para el Hospital estudiado, se puede concluir que al usar la regla $L P T$, se programan en 19 días las cirugías de un mes. Esto implica que, en los once días restantes, se podrán realizar cirugías adicionales. Por otro lado, esta programación permite reducir el tiempo de espera, para una cirugía, en un promedio de 6,2 días. En escenarios donde el tiempo de oportunidad sea superior a un mes, ambos indicadores podrían mejorar sustancialmente. Finalmente, la tasa de ocupación encontrada es del $77,28 \%$, con un coeficiente de variación de 20,66 \%. Esto significa un incremento del $26,72 \%$, con respecto a la tasa de ocupación actual del Hospital.

Ahora bien, debido a las particularidades de cada servicio quirúrgico, la calidad de las soluciones debe medirse con respecto a la situación real del hospital. En ese orden de ideas, no es posible hacer comparaciones directas con los resultados obtenidos en otros estudios. Sin embargo, las mejoras porcentuales encontradas en este proyecto son cercanas a las reportadas por otros autores. En términos del volumen de atención semanal, se encontró una mejora del $6,45 \%$, mientras que Smith y Litvak reportaron mejoras del $4 \%$ y $5 \%$, respectivamente $(9,27)$. Por otro lado, al analizar los porcentajes de utilización, Bender et al. reportan una mejora del $12 \%$ y Sandbaek, una mejora del
$6 \%(28,29)$. Este estudio encontró una mejora del $26,72 \%$ con respecto a la utilización actual del servicio.

Estos resultados aportan evidencia para la discusión de un problema localmente relevante. De acuerdo con Velasco et al., en Colombia no se han hecho aproximaciones sistemáticas para el uso de la investigación de operaciones en el sector salud (17). Adicionalmente, las investigaciones conducidas en otros contextos no tienen impacto en la comunidad clínica (2). En ese sentido, autores como Hulshof et al. y Dobrzykowski et al. afirman que uno de los principales retos es conducir investigaciones en las que participen de manera activa los responsables del proceso en el Hospital $(5,6)$. Al probar el impacto en dos indicadores críticos, este estudio busca promover el uso de herramientas formales para soportar la toma de decisiones en el sector salud, por medio de investigaciones interdisciplinarias.

Tres extensiones a este trabajo pueden ser de interés. Por un lado, no se estudió la programación de recursos que pueden ser limitantes. En ese orden de ideas, puede analizarse el desempeño del algoritmo en un problema que incluya restricciones de disponibilidad e incompatibilidad de los procedimientos. Por otro lado, de acuerdo con Schultz y Velásquez et al., la inclusión de la variabilidad, asociada con la duración 
de los tiempos quirúrgicos, cumple un papel fundamental en la implementación de los resultados $(16,30)$. Con el fin de capturar esta variabilidad, se puede modelar la duración de las cirugías como una variable aleatoria. Finalmente, se considera relevante explorar el impacto de técnicas más elaboradas para el problema de optimización. De acuerdo con Baesler et al., el uso de meta heurísticas puede mejorar considerablemente la calidad de las soluciones (31).
Agradecimientos

Al personal del Departamento de Cirugía del Hospital Universitario Mayor - Méderi por su disposición y ayuda durante la realización de este estudio.

\section{Descargos de responsabilidad}

Los autores declaran que no tienen ningún conflicto de interés. Este estudio no ha recibido financiación diferente a la interna por parte de las instituciones ejecutoras. Proyecto "Impacto al implementar estrategias de ordenamiento en la programación de cirugías", código 0006687.

\section{Referencias}

1. Fernández-García JJ. La hora de la gestión de las operaciones: una visita guiada. Rev Sedisa S xxI. 2009;12:70-5.

2. Brailsford S, Vissers J. OR in healthcare: A European perspective. Eur J Oper Res 2011;212(2):223-34.

3. Denton B, Viapiano J, Vogl A. Optimization of surgery sequencing and scheduling decisions under uncertainty. Health Care Manag Sci 2007;10(1):13-24.

4. Gordon T, Paul S, Lyles A, Fountain J. Surgical unit time utilization review: resource utilization and management implications. J Med Syst 1988;12(3):169-79.

5. Dobrzykowski D, Saboori Deilami V, Hong P, Kim SC. A structured analysis of operations and supply chain management research in healthcare (1982-2011). Int J Prod Econ 2014;147(2014):514-30.

6. Hulshof PJH, Kortbeek N, Boucherie RJ, Hans EW, Bakker PJM. Taxonomic classification of planning decisions in health care: a structured review of the state of the art in OR/MS. Heal Syst 2012;1(2):129-75.

7. Lynch R. Economic considerations, efficiency and design. En: Kaye AD, Fox CJ, Urman RD, eds. Operating Room Leadership and Management. 1. a ed. New York: Cambridge University Press; 2012. p. 39-45.

8. Litvak E. Managing Patient Flow in Hospitals: Strategies and Solutions. 2. ${ }^{\text {a }}$ ed. Oakbrook Terrace, Illinois: Joint Commission Resources; 2010.

9. Smith CD, Spackman T, Brommer K, Stewart MW, Vizzini M, Frye J, et al. Re-engineering the operating room using variability methodology to improve health care value. J Am Coll Surg 2013;216(4):559-68; discussion 568-70.

10. Gabriele A, Wang J, Yang K. Operating room adjusted utilization studynull. Int J Lean Six Sigma may2015;6(2):111-37.

11. Chana P, Bunrs E, Arora S, Darzi A, Faiz O. A Systematic Review of the Impact of Dedicated Emergency Surgical Services on Patient Outcomes. Ann Surg 2015;263(1):20-7.

12. Astaraky D, Patrick J. A simulation based approximate dynamic programming approach to multi-class, multi-resource surgical scheduling. Eur J Oper Res 2015;245(1):309-19.

13. Jiménez A, Amaya C, Velasco N. Estrategias de programación de salas de cirugía: Un caso de aplicación en un hospital de Bogotá. Bogotá; 2008. 
Reglas de despacho en la programación de procedimientos quirúrgicos electivos: impacto en los indicadores de ocupación y oportunidad

14. Díaz-López LP, Fuquen-Fraile L, Barrera D, González-Neira EM, García-Herreros LG, Suárez DR. Control de la variabilidad en la programación de pacientes electivos en salas de cirugía. Gerenc y Políticas Salud 2015;14(28):78-87.

15. Alonso E, Tobón Á, Andrea P, Restrepo V, Villegas JG. Analyzing the Capacity of the IPS Universitaria Surgery Service Via Integer Programming. Rev Ing Biomédica 2015;9(18):127-32.

16. Velásquez-Restrepo PA, Rodríguez-Quintero AK, Jaén-Posada JS. Aproximación metodológica a la planificación y a la programación de las salas de cirugía: una revisión de la literatura. Rev Gerenc y Polit Salud 2012;2(24):249-66.

17. Velasco N, Barrera D, Amaya CA. Logística hospitalaria: Lecciones y retos para Colombia. En: Bernal O, Gutiérrez C, eds. La salud en Colombia. Logros, retos y recomendaciones. Bogotá: Uniandes; 2012. p. 309-43.

18. Amaya CA, Beaulieu M, Landry S, Rebolledo C, Velasco N. Potenciando la contribución de la logística hospitalaria: tres casos, tres trayectorias. Manag Int 2010;14(4):85.

19. Saadouli H, Jerbi B, Dammak A, Masmoudi L, Bouaziz A. A stochastic optimization and simulation approach for scheduling operating rooms and recovery beds in an orthopedic surgery department. Comput Ind Eng 2015;80:72-9.

20. Xiang W, Yin J, Lim G. A short-term operating room surgery scheduling problem integrating multiple nurses roster constraints. Artif Intell Med 2015;63(2):91-106.

21. Dexter F, Macario A, Traub RD, Hopwood M, Lubarsky DA. An operating room scheduling strategy to maximize the use of operating room block time: computer simulation of patient scheduling and survey of patients' preferences for surgical waiting time. Anesth Analg 1999;89(1):7-20.

22. Lawrence S. Heuristic, optimal, static, and dynamic schedules when processing times are uncertain. J Oper Manag 1997;15(1):71-82.

23. Pinedo ML. Scheduling: Theory, Algorithms and Systems. 4. ${ }^{a}$ ed. New York: Springer Science \& Business Media; 2012.

24. Li WM, L. Mitchell V, R. Nault B, Brind D. Operating Room Scheduling and Adaptive Control Using a Priority First Fit Decreasing Heuristic. Eng Manag Res 2015;4(2):46-53.

25. Zheng F, Luo L, Zhang E. NF-based algorithms for online bin packing with buffer and bounded item size. J Comb Optim 2015;30(2):360-9.

26. Blake JT, Donald J. Mount Sinai Hospital Uses Integer Programming to Allocate Operating Room Time. Interfaces 2002;32(2):63-73.

27. Litvak E. Optimizing Patient Flow by Managing Its Variability. In: Berman S, ed. From Front Office to Front Line: Essential Issues for Health Care Leaders. 1. ${ }^{a}$ ed. Oakbrook Terrace, Illinois: Joint Commission Resources; 2005. p. 91-112.

28. Bender JS, Nicolescu TO, Hollingsworth SB, Murer K, Wallace KR, Ertl WJ. Improving operating room efficiency via an interprofessional approach. Am J Surg 2015;209(3):447-50.

29. Sandbaek BE, Helgheim BI, Larsen OI, Fasting S. Impact of changed management policies on operating room efficiency. BMC Health Serv Res 2014;14(1):224.

30. Schultz J. Variability based surgical scheduling: a simulation approach. Proceedings of the 2014 Winter Simulation Conference. 2014. p. 1353-64; Savannah.

31. Baesler F, Gatica J, Correa R. Simulation Optimisation for Operating Room Scheduling. Int J Simul Model 2015; 4(2):215-26. 